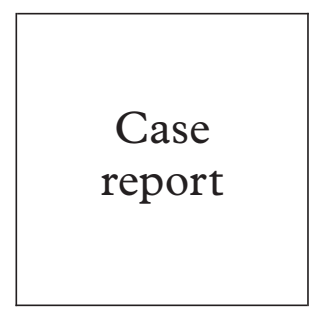

\title{
Graded challenge in an aciclovir allergic patient
}

\author{
M Kawsar, J M Parkin, G Forster
}

An immunocompetent woman presented with a hypersensitivity skin reaction following suppressive therapy with aciclovir for recurrent culture proved genital herpes simplex virus infection. She developed a similar reaction when treatment was changed to famciclovir. Without antiviral suppression her recurrences were frequent and distressing. Graded challenge was performed and she became tolerant to aciclovir. She successfully continued suppressive therapy for 1 year with no further hypersensitivity reactions or recurrences.

(Sex Transm Inf 2001;77:204-205)

Keywords: aciclovir; genital herpes; graded challenge

\section{Case report}

A 48 year old Afro-Caribbean woman presented in 1997 with recurrent culture proved genital herpes simplex virus (HSV) type 2 infection every 2-4 weeks since 1995 . Topical aciclovir had not controlled the attacks and she commenced suppressive therapy with aciclovir $400 \mathrm{mg}$ twice daily. On this regimen she had no further episodes of HSV. Eight months later she developed a periumbilical erythematous maculopapular rash and generalised itching. She was otherwise well and taking no other medication. An allergic drug reaction was suspected and the rash resolved completely within days of stopping aciclovir. However, she developed two recurrences of HSV within the next 6 weeks. She was therefore commenced on famciclovir $250 \mathrm{mg}$ twice daily. Three weeks later she again developed an itchy maculopapular rash, this time affecting her arms and legs. As before there was no concurrent drug therapy. The rash resolved within a few days of cessation of famciclovir. Two months later, the patient reintroduced famciclovir. The same rash returned within 4 days and resolved promptly on stopping the drug. At no stage were there signs of mucosal involvement or systemic allergic reaction. The patient had a history of drug allergies with rashes to aspirin, penicillins, cephalosporins, and pethidine. She also had contact sensitivity to soaps and detergents. She was atopic with mild asthma and seasonal rhinitis. There was no other history of note. Investigations revealed normal full blood count and biochemistry with no evidence of autoimmune disease or immunodeficiency.

The temporal relation between the initiation and/or cessation of the drugs and the development of the rash suggested a hypersensitivity reaction to the antiviral drugs. A cross reaction between famciclovir and aciclovir may occur because of structural similarity, although famciclovir has been successfully used in patients with aciclovir allergy. ${ }^{1}$ The timing of the reaction and clinical appearance were suggestive of a cellular rather than IgE mediated reaction. The history and examination findings were central to this diagnosis. There is no commercial serological assay for aciclovir specific IgE. Skin (prick or patch) testing can be done with solutions of drugs, but false positives are common and the predictive value of a negative test is largely unknown.

Without therapy the HSV attacks were frequent and distressing. The patient requested suppressive therapy and was offered graded challenge to aciclovir with the aim of inducing tolerance to the drug. The regimen used doubling doses from 100th of the required dose of aciclovir (table 1). Two doses were given daily, 6 hours apart under oral antihistamine cover. The challenge was performed in a hospital day ward with physician cover and full resuscitation facilities. Pulse and blood pressure were monitored regularly for 2 hours after each dosing.

She continued suppressive treatment with aciclovir $200 \mathrm{mg}$ three times daily for a year with no HSV recurrence. The skin rash remained quiescent apart from a small erythematous area above the eyebrows at 3 months of treatment. This resolved within a week on reduction of aciclovir to $200 \mathrm{mg}$ twice daily and antihistamine therapy. Suppressive therapy was stopped in September 1999 with no further episodes of HSV.

The management of patients with drug allergies generally involves avoidance of the drug. When the drug needs to be given there are two options. In IgE mediated reactions rapid desensitisation is used. This involves the rapid administration of increasing doses of the drug from very small amounts (usually $10^{-6}$ of the therapeutic dose) at 15-30 minute intervals to alter effector cell responses. Successful rapid desensitisation using oral aciclovir has been reported in an AIDS patient who developed

\section{Table 1 Dosage regimen}

\begin{tabular}{lr}
\hline Day & \multicolumn{1}{c}{ Dose } \\
\hline 1 & $2 \mathrm{mg}$ \\
Aciclovir suspension & $4 \mathrm{mg}$ \\
2 & $8 \mathrm{mg}$ \\
Aciclovir suspension & $16 \mathrm{mg}$ \\
3 & $32 \mathrm{mg}$ \\
Aciclovir suspension & $64 \mathrm{mg}$ \\
4 & $100 \mathrm{mg}$ \\
Aciclovir tablets & $200 \mathrm{mg}$ \\
5 & $200 \mathrm{mg}$ \\
Aciclovir tablets & $200 \mathrm{mg}$ \\
& $200 \mathrm{mg}$ \\
\hline
\end{tabular}

Accepted for publication 8 March 2001 
angioedema with aciclovir therapy. ${ }^{2}$ Graded challenge is used in non- $\operatorname{IgE}$ mediated reactions and involves administration of small doses of the drug, starting at approximately $10^{-2}$ of the therapeutic dose, increasing over days or weeks to confer clinical tolerance. ${ }^{3}$ This technique has been used with a success rate of approximately $75 \%$ in HIV positive patients with allergic skin reactions to sulphamethoxazole-trimethoprim (co-trimoxazole). This is the first report of graded challenge to aciclovir in an immunocompetent individual. The graded challenge approach to non- $\operatorname{IgE}$ mediated drug hypersensitivities (which may account for up to $25 \%$ of all drug reactions) is not widely utilised and yet may be useful in other cases of allergic drug reactions where there are no appropriate therapeutic alternatives.

1 Bayrou O, Gaouar H, Leynadier F. Famciclovir as a possible alternative treatment in some cases of allergy to aciclovir. Contact Dermatitis 2000;42:42.

2 Henry RE, Wegmann JA, Hartle JE, et al. Successful oral aciclovir desensitization. Ann Allergy 1993;70:386-8.

3 Joint Task Force on Practice parameters. Disease managejoint Task Force on Practice parameters. Disease manage-
ment of drug hypersensitivity: a practical parameter. Ann Allergy Asthma Immunol 1999;83:666-95.

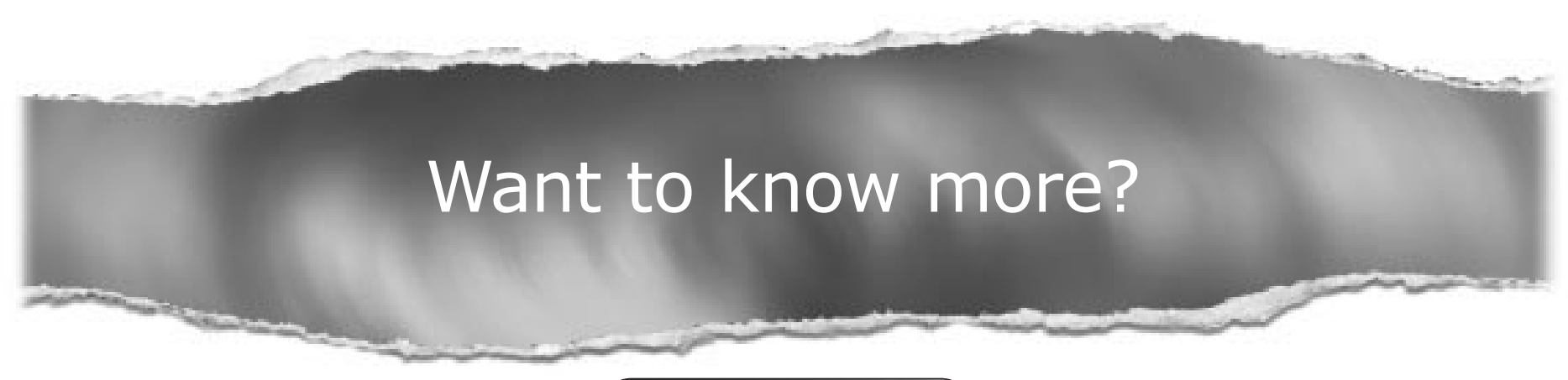

Data supplements

Limited space in printed journals means that interesting data and other material are often edited out of articles; however, limitless cyberspace means that we can include this information online. Look out for additional tables, references, illustrations.

www.sextransinf.com 